\title{
Erratum
}

\section{Synthesis of 1-Chloroalkynes from Alkynylsilanes Using Trichloroisocyanuric Acid as Chlorinating Agent}

Mie Højer Vilhelmsen, Asbjørn Sune Andersson, Mogens Brøndsted Nielsen* Synthesis 2009, 1469.

When revisiting the reaction conditions and the product composition of the previously reported chlorination reaction, we found that the reaction products are, in the case of unsubstituted and donor-substituted arylalkynes $(\mathrm{R}=\mathrm{H}$, $\mathrm{OMe}, t$-Bu), not exclusively the reported chloroalkynes but to a large extent also chlorinated ketones.

Corrected products and yields under various conditions for the chlorination of $p-\mathrm{RC}_{6} \mathrm{H}_{4} \mathrm{C} \equiv \mathrm{CSiMe}_{3}$ using trichloroisocyanuric acid (TCCA) and silver nitrate are provided here. These should replace Scheme 2 and Table 1 in the original paper. We apologize for the mistake and for any inconvenience it may have caused.

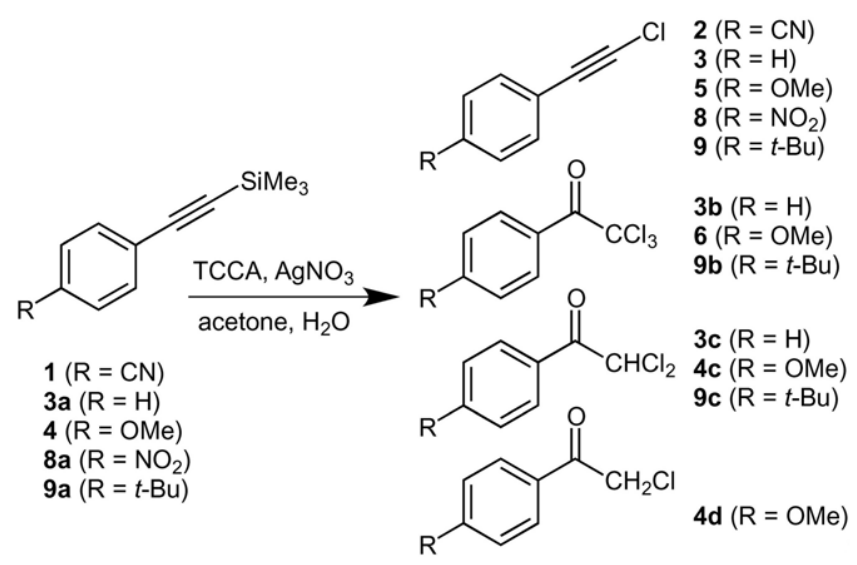

Scheme 2

Table 1 Corrected Products and Yields Obtained from Chlorination of $p-\mathrm{RC}_{6} \mathrm{H}_{4} \mathrm{C} \equiv \mathrm{CSiMe}_{3}$

\begin{tabular}{|c|c|c|c|c|c|}
\hline $\mathrm{R}$ (starting material) & Scale $^{\mathrm{a}}$ & $\mathrm{TCCA}^{\mathrm{b}}$ & $\mathrm{AgNO}_{3}{ }^{\mathrm{c}}$ & Conditions $^{\mathrm{d}}$ & Product yields $(\%)$ \\
\hline $\mathrm{CN}(\mathbf{1})$ & 1.53 & 1.03 & 0.10 & $0^{\circ} \mathrm{C}$ for $5 \mathrm{~h}$ & $2(93)$ \\
\hline $\mathrm{CN}(\mathbf{1})$ & 1.59 & 1.03 & 0.19 & $\mathrm{H}_{2} \mathrm{O}$ (19 equiv); $0{ }^{\circ} \mathrm{C}$ for $30 \mathrm{~min}$, then $40^{\circ} \mathrm{C}$ for $24 \mathrm{~h}$ & $2(84)$ \\
\hline $\mathrm{CN}(\mathbf{1})$ & 1.54 & 1.07 & 0.20 & $\mathrm{H}_{2} \mathrm{O}$ (20 equiv); $0{ }^{\circ} \mathrm{C}$ for $30 \mathrm{~min}$, then $\mu \mathrm{w}$ for $5 \mathrm{~min}$ & $2(98)$ \\
\hline $\mathrm{H}(\mathbf{3 a})$ & 1.82 & 1.00 & 0.10 & $0{ }^{\circ} \mathrm{C}$ for $5 \mathrm{~h}$, then r.t. for $19 \mathrm{~h}$ & $\mathbf{3 b}(23)$ \\
\hline $\mathrm{H}(\mathbf{3 a})$ & 1.02 & 1.08 & 0.21 & $0^{\circ} \mathrm{C}$ for $30 \mathrm{~min}$, then reflux for $20 \mathrm{~h}$ & $\mathbf{3}(7) ; \mathbf{3 b}(34) ; \mathbf{3 c}(8)$ \\
\hline $\mathrm{H}(\mathbf{3 a})$ & 1.02 & 1.09 & 0.20 & $0^{\circ} \mathrm{C}$ for $30 \mathrm{~min}$, then $\mu \mathrm{w}$ for $5 \mathrm{~min}$ & $\mathbf{3}(<20) ; \mathbf{3 b}(8) ; \mathbf{3 c}(10)$ \\
\hline $\mathrm{OMe}(\mathbf{4})$ & 1.54 & 1.00 & - & $\begin{array}{l}\mathrm{Bu}_{4} \mathrm{NF}\left(1 \mathrm{M} \text { soln in } \mathrm{THF} ; 0.68 \text { equiv); } 0{ }^{\circ} \mathrm{C} \text { for } 70 \mathrm{~min} \text {, }\right. \\
\text { then r.t. for } 30 \mathrm{~min}\end{array}$ & $5(11) ; 6(25)$ \\
\hline $\mathrm{OMe}(\mathbf{4})$ & 1.59 & 1.07 & 0.09 & $0{ }^{\circ} \mathrm{C}$ for $30 \mathrm{~min}$, then $40-50{ }^{\circ} \mathrm{C}$ for $72 \mathrm{~h}$ & $\mathbf{6}(31) ; \mathbf{4 c}(27) ; \mathbf{4 d}(21)$ \\
\hline $\mathrm{OMe}(\mathbf{4})$ & 1.03 & 1.07 & 0.20 & $0^{\circ} \mathrm{C}$ for $30 \mathrm{~min}$, then reflux for $20 \mathrm{~h}$ & $6(29) ; 4 \mathbf{c}(37)$ \\
\hline $\mathrm{OMe}(4)$ & 1.03 & 1.08 & 0.19 & $0^{\circ} \mathrm{C}$ for $30 \mathrm{~min}$, then $\mu \mathrm{w}$ for $5 \mathrm{~min}$ & $6(26) ; 4 \mathbf{c}(24)$ \\
\hline $\mathrm{NO}_{2}(\mathbf{8 a})$ & 1.38 & 1.10 & 0.21 & $\mathrm{H}_{2} \mathrm{O}$ (20 equiv); $0{ }^{\circ} \mathrm{C}$ for $30 \mathrm{~min}$, then $40^{\circ} \mathrm{C}$ for $24 \mathrm{~h}$ & $8(50)$ \\
\hline $\mathrm{NO}_{2}(\mathbf{8 a})$ & 1.40 & 1.12 & 0.21 & $\mathrm{H}_{2} \mathrm{O}$ (20 equiv); $0{ }^{\circ} \mathrm{C}$ for $5 \mathrm{~min}$, then $\mu \mathrm{w}$ for $5 \mathrm{~min}$ & $8(42)$ \\
\hline $\mathrm{NO}_{2}(\mathbf{8 a})$ & 1.00 & 1.12 & 0.20 & $0^{\circ} \mathrm{C}$ for $30 \mathrm{~min}$, then reflux for $20 \mathrm{~h}$ & $8(76)$ \\
\hline $\mathrm{NO}_{2}(\mathbf{8 a})$ & 1.00 & 1.10 & 0.20 & $0^{\circ} \mathrm{C}$ for $30 \mathrm{~min}$, then $\mu \mathrm{w}$ for $5 \mathrm{~min}$ & $8(>99)$ \\
\hline$t-\mathrm{Bu}(\mathbf{9 a})$ & 1.08 & 1.13 & 0.23 & $\mathrm{H}_{2} \mathrm{O}$ (20 equiv); $0{ }^{\circ} \mathrm{C}$ for $30 \mathrm{~min}$, then $40{ }^{\circ} \mathrm{C}$ for $30 \mathrm{~min}$ & $9(43) ; 9 b(57)$ \\
\hline$t$-Bu $(9 \mathbf{a})$ & 0.84 & 1.11 & 0.20 & $\mathrm{H}_{2} \mathrm{O}$ (20 equiv); $0{ }^{\circ} \mathrm{C}$ for $5 \mathrm{~min}$, then $\mu \mathrm{w}$ for $5 \mathrm{~min}$ & $\mathbf{9}(26) ; \mathbf{9 b}(58)$ \\
\hline$t$-Bu $(\mathbf{9 a})$ & 0.97 & 1.13 & 0.21 & $0^{\circ} \mathrm{C}$ for $30 \mathrm{~min}$, then reflux for $20 \mathrm{~h}$ & $9 \mathbf{b}(34) ; \mathbf{9 c}(23)$ \\
\hline$t$-Bu $(\mathbf{9 a})$ & 1.02 & 1.08 & 0.20 & $0{ }^{\circ} \mathrm{C}$ for $30 \mathrm{~min}$, then $\mu \mathrm{w}$ for $5 \mathrm{~min}$ & $9 \mathbf{b}(19) ; 9 \mathbf{c}(<13)$ \\
\hline
\end{tabular}

${ }^{a}$ Amount of $p-\mathrm{RC}_{6} \mathrm{H}_{4} \mathrm{C} \equiv \mathrm{CSiMe}_{3}$ in mmol.

${ }^{b}$ Molar equivalents of TCCA relative to $p-\mathrm{RC}_{6} \mathrm{H}_{4} \mathrm{C} \equiv \mathrm{CSiMe}_{3}$.

${ }^{c}$ Molar equivalents of $\mathrm{AgNO}_{3}$ relative to $p-\mathrm{RC}_{6} \mathrm{H}_{4} \mathrm{C} \equiv \mathrm{CSiMe}_{3}$.

${ }^{\mathrm{d}} \mu \mathrm{w}=$ microwave heating at $150{ }^{\circ} \mathrm{C}$. 